\title{
Stress induced by messenger ribonucleic acid (RNA) vaccination may reveal acute adrenal insufficiency
}

\author{
C. Mariette $\mathbb{i}^{1} \cdot$ J. Lavaud ${ }^{1} \cdot$ V. Descamps ${ }^{1}$
}

Received: 5 September 2021 / Accepted: 11 September 2021 / Published online: 20 September 2021

(c) The Author(s), under exclusive licence to Springer Science+Business Media, LLC, part of Springer Nature 2021

Dear Editor,

Vaccines are well known to activate the immune system and induce physical stress.

Messenger ribonucleic acid (mRNA) vaccines had never been used in humans before the coronavirus disease 2019 (COVID-19) pandemic.

The principle is to inject the messenger RNA coding for the severe acute respiratory syndrome coronavirus-2 (SARS-CoV2) Spike protein, a protein that allows the virus to enter and infect cells. The synthesis of the Spike protein by human cells induces the activation of adaptive immunity and creates a stress for the organism which may require a higher production of cortisol.

L. Katznelson and M. Gadelha from the Pituitary Society studied how glucocorticoid replacement in patients was managed with adrenal insufficiency receiving a COVID-19 vaccine [1]. Most clinicians plan to maintain the current glucocorticoid dose with vaccine administration, and to increase glucocorticoid dose in case of fever, and just under half in case of arthralgias and myalgias [1].

Routine vaccinations are known as a precipitant of adrenal crisis in chronic adrenal insufficiency [2] but data on RNA vaccination are poor.

We would like to draw your attention to the case of an 87year-old patient followed for an acro-lentiginous melanoma of the left foot with subcutaneous and lymph node metastases, treated by immunotherapy (Ipilimumab and Nivolumab) and radiotherapy. A first dose of mRNA COVID 19 vaccine (Pfizer/BioNTech) at the start of her anti-cancer treatment was performed without any complication. After four infusions of Ipilimumab and Nivolumab, she received her 2 nd injection of mRNA vaccine. Soon after vaccination, anorexia, asthenia, and vomiting appeared. On the 3rd day,

\footnotetext{
V. Descamps

vincent.descamps@aphp.fr

1 Bichat Hospital, Paris, France
}

she was found unconscious at home in deep hypoglycemia. On arrival at the hospital, the patient was febrile at $38^{\circ} \mathrm{C}$, she presented hyponatremia and hyperkalemia.

Low levels of cortisol ( $98 \mathrm{nmol} / \mathrm{L}$ on the morning) and adrenocorticotropin $(\mathrm{ACTH}<5 \mathrm{pg} / \mathrm{mL})$ revealed central adrenal insufficiency. TSH was normal. Pituitary MRI confirmed pan pituitary inflammation. Hydrocortisone supplementation was started allowing rapid resolution of symptoms. The retained diagnosis was a central adrenal insufficiency secondary to immunotherapy, revealed by the stress and immune reaction induced by mRNA Covid19 vaccine. Pfizer/BioNTech vaccine is broadly considered safe but may rarely reveal or induce immune disorders such as thyroiditis [3]. Clinicians must be aware of these rare events.

Many guidelines on the management of the COVID 19 crisis in patients with adrenal insufficiency have been written recently but they do not address clearly the subject of vaccination. The question of increasing hydrocortisone supplementation before, at the time of vaccination or in the days following should be discussed by endocrinological societies and be the subject of recommendations.

\section{Data and material availability}

Not applicable.

\section{Code availability}

Not applicable

Acknowledgements Thanks to the Dermatology Department of Bichat Hospital.

Funding This research did not receive any specific grant from any funding agency in the public, commercial or not-for-profit sector.

\section{Compliance with ethical standards}

Conflict of interest The authors declare no competing interests. 
Consent for publication Consent has been obtained from the patient for publication.

Consent to participate Consent has been obtained from the patient for participation.

Ethical approval Not applicable.

Publisher's note Springer Nature remains neutral with regard to jurisdictional claims in published maps and institutional affiliations.

\section{References}

1. L. Katznelson, M. Gadelha, Glucocorticoid use in patients with adrenal insufficiency following administration of the COVID-19 vaccine: a pituitary society statement. Pituitary. 24(2), 143-145 (2021)

2. A. Major, K. Chacko, Routine vaccinations as a precipitant of adrenal crisis in adrenal insufficiency. Intern. Med. J. 48(3), 360-361 (2018)

3. J. Schimmel, E.L. Alba, A. Chen, M. Russell, R. Srinath, Letter to the Editor: Thyroiditis and Thyrotoxicosis After the SARS-CoV-2 mRNA Vaccine. Thyroid 31(9), 1440 (2021). 